\title{
Estudo das dimensões transversais da face, em telerradiografias póstero-anteriores em indiví- duos respiradores bucais com oclusão normal e má oclusão Classe I de Angle
}

\author{
Rosemári Fistarol DANIEL*, Orlando TANAKA**, Luiz Renato C. ESSENFELDER***
}

\begin{abstract}
Resumo
A respiração bucal vem sendo estudada há mais de 100 anos, na tentativa de entender os reais envolvimentos com as más oclusões. Este estudo tem como objetivo avaliar as dimensões transversais da face em 60 crianças, 32 do gênero feminino e 28 do masculino, com idades entre 6 anos e 1 mês e 8 anos e 2 meses, com má oclusão Classe I de Angle, com respiração nasal ou bucal. Foram selecionadas aleatoriamente 60 telerradiografias em norma frontal pósteroanterior e realizados os traçados cefalométricos para a obtenção das 26 variáveis de interesse. A avaliação do padrão respiratório de cada criança foi realizada por meio de um exame clínico específico. Após o diagnóstico do modo respiratório, a amostra foi dividida em subgrupos de oclusão normal e respiração nasal (14 crianças), oclusão normal e respiração bucal (11 crianças), Classe I de Angle e respiração nasal (6 crianças), Classe I de Angle e respiração bucal (29 crianças). Os resultados encontrados indicam que não existem diferenças estatisticamente significativas nas dimensões transversais da face entre os grupos. Além disso, não foi encontrada correlação entre o modo respiratório bucal e a mordida cruzada dentária posterior.
\end{abstract}

Palavras-chave: Respiração bucal. Telerradiografias póstero-anteriores. Diagnóstico.

\section{INTRODUÇÃO E FUNDAMENTOS TEÓRICOS}

Os estudos verificados na literatura indicam, de maneira geral, que a alteração da função respiratória nasal para bucal, principalmente durante a fase de crescimento facial, poderia causar efeitos indesejáveis sobre os tecidos faciais dento-esqueléticos, modificar a função muscular mastigatória e peribucal e também provocar alterações trans- versais na região média da face.

Estas questões, são o resultado de mais de um século de indagações e controvérsias, visto que, em 1873, Tomes $^{41}$ descrevia, pela primeira vez, as características da "face adenoideana" e relatava que crianças com respiração bucal, freqüentemente, apresentavam arcos dentários estreitos - em forma de "V". Esta última, te-

* Mestre em Odontologia (Ortodontia) pela PUCPR.

** Doutor em Odontologia - Ortodontia FO-UFRJ - Professor Titular - CCBS - Professor do Curso de Mestrado em Odontologia - Ortodontia da PUCPR.

*** Doutor em Odontologia - Ortodontia UNICAMP Piracicaba. 
ria sido interpretada por Kingsley (1888 apud O'RYAN ${ }^{29}$, 1982) como uma característica congênita, não relacionada com a respiração bucal. Para Angle ${ }^{1}$ a respiração bucal era considerada como a causa mais importante no desenvolvimento da má oclusão. Por sua vez, Emslie et al. ${ }^{8}$, afirmaram que deveria haver uma associação entre a predisposição anatômica e a obstrução nasal. Em contrapartida, para Gwynne-Evans e Ballard ${ }^{15}$ a respiração bucal não produziria deformidades nas arcadas e, conseqüentemente, não provocaria más oclusões ou faces adenoideanas.

É comum a descrição da face adenoideana associada a características tais como postura de boca aberta, nariz estreito com narinas pequenas, lábio superior curto, arco maxilar em forma de "V", palato profundo ou ogival, incisivos superiores protruídos e uma relação oclusal de Classe II ${ }^{42}$. Porém, o que se pode observar é que nem todos os indivíduos que apresentam estas características são efetivamente respiradores bucais e, nem todos os respiradores bucais possuem essencialmente tais características ${ }^{25,39}$.

Para Hartsook ${ }^{17}$, Gwynne-Evans ${ }^{14}$, GwynneEvans e Ballard ${ }^{15}$ e Hawkins ${ }^{18}$ o desequilíbrio muscular existente no respirador bucal, causado pela musculatura dos lábios e das bochechas externamente e, internamente pela língua, produziria pressões alteradas sobre o arco dentário superior, provocando o seu estreitamento.

$\mathrm{E}$ as alterações transversais, decorrentes da respiração bucal, referidas por muitos estudiosos como atresias maxilares, arcos dentários em forma de ' $V$ ', mordidas cruzadas posteriores? Estariam elas presentes em indivíduos com crescimento facial ântero-posterior harmonioso? No estudo da respiração bucal versus má oclusão, o que parece importante é dissociar as relações interoclusais nos três planos do espaço. Desta maneira, as más oclusões ântero-posteriores representadas pelas Classes II e III de Angle já apresentam desarmonias esqueléticas específicas. Em indivíduos com crescimento predominantemente vertical os efeitos nocivos da respiração bucal são clínica e cefalometricamente mais evidentes.

Outra questão a ser considerada em relação às alterações transversais é a localização das estruturas da face envolvidas, esquelética e/ou dentária, e de como é feito rotineiramente o diagnóstico. Observa-se, de maneira geral, que este geralmente é realizado clinicamente ou em modelos de gesso, sem a utilização de outro elemento complementar que possibilitaria uma avaliação mais profunda das estruturas afetadas.

Assim os objetivos deste estudo são: verificar se há diferenças nas dimensões transversais da face de indivíduos com oclusão normal e Classe I de Angle, com respiração bucal e com respiração nasal; e ainda verificar se há nesta amostra correlação entre respiração bucal e mordida cruzada dentária posterior.

\section{MATERIAL E MÉTODO}

Foram estudadas 60 telerradiografias pósteroanteriores de crianças com má oclusão Classe I de Angle e Oclusão Normal, brasileiras, leucodermas, 28 do gênero masculino e 32 do gênero feminino. A faixa de idade da amostra era de 6 anos e 1 mês a 8 anos e 2 meses. A avaliação do modo respiratório foi clínica, de acordo com o proposto por Wagnitz ${ }^{43}$.

Após realizado o diagnóstico do modo respiratório a amostra foi dividida em 4 subgrupos distintos:

1A) Oclusão Normal respiração nasal, 14 crianças,

1B) Oclusão Normal e respiração bucal, 11 crianças,

2A) Classe I e respiração nasal, 6 crianças e,

2B) Classe I e respiração bucal, 29 crianças.

O critério utilizado para classificar esta amostra em fase de dentição mista, foi considerando que o primeiro molar superior deveria apresentar-se com a cúspide mésio-vestibular ocluindo no sulco mésio vestibular do primeiro molar inferior, semelhante à Classe I de Angle; ou com plano terminal dos segundos molares decíduos em degrau mesial 
ou reto, e com os caninos decíduos em relação ântero-posterior normal, isto é, canino superior decíduo ocluindo no espaço primata inferior.

\section{TRAÇADO CEFALOMÉTRICO} Linhas Cefalométricas

Linha Esfenoidal Horizontal (LEH) - obtida pela união dos pontos esfenóide ( $\mathrm{Sph}$ ) direito e esquerdo (Fig 1).
Linha Média Sagital Verdadeira (LMV) - linha com sentido vertical traçada, ortogonalmente, a partir da LEH, a qual secciona o ponto Nc (Crista galli), estendendo-se além do rebordo inferior da mandíbula (Fig. 1).

\section{Grandezas Lineares}

Esfenóide Total (SphT), direito (SphD) e esquerdo (SphE); Zigomático Total (ZygT), direito

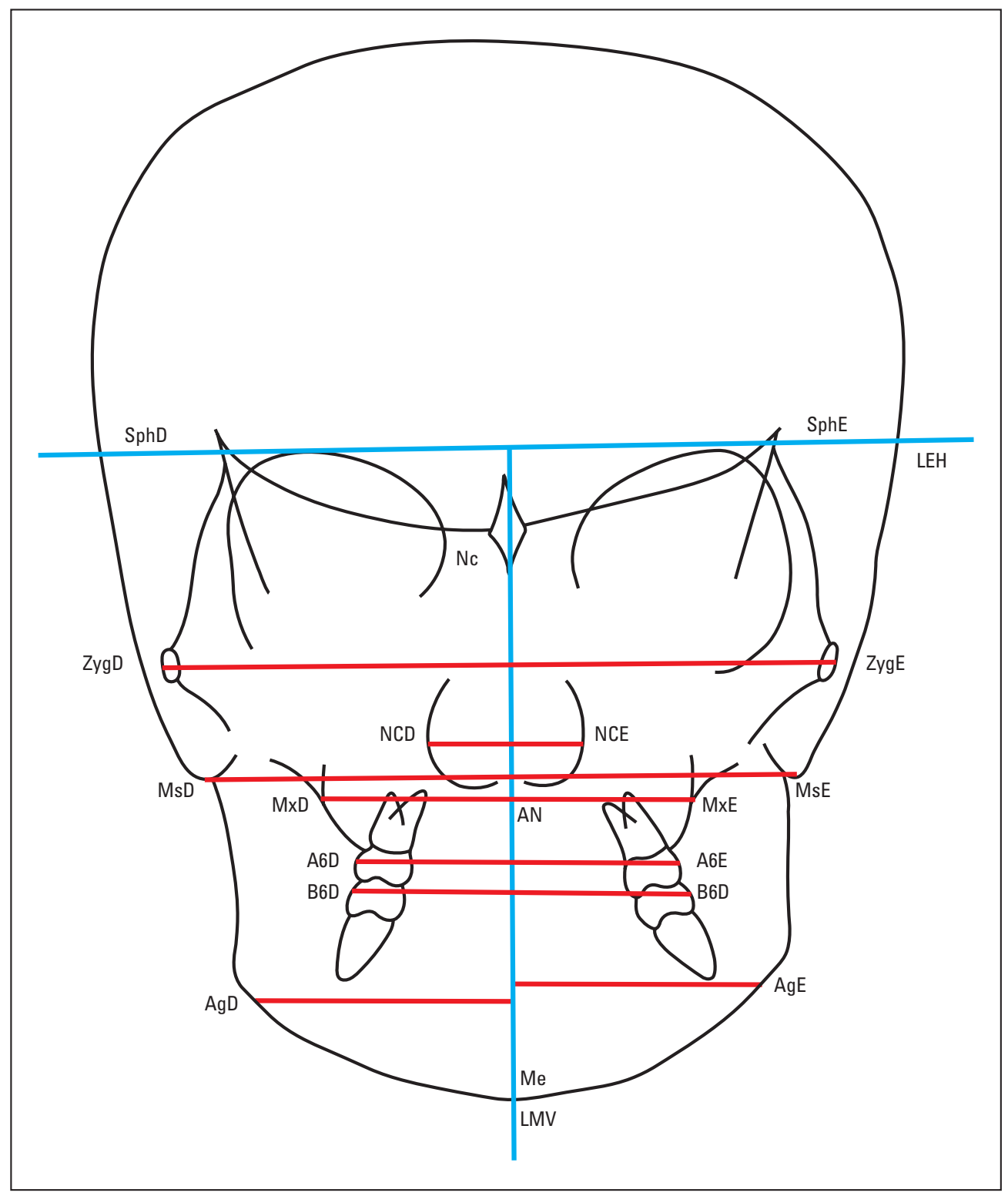

FIGURA 1 - Pontos cefalométricos, linha esfenoidal horizontal (LEH), linha mediana sagital verdadeira (LMV). Grandezas transversais: Esfenóide total, zigomático total, mastóide total, cavidade nasal total, maxila total, molar superior total, molar inferior total, mandíbula total, espinha nasal anterior e mento. 
(ZygD) e esquerdo (ZygE);. Mastóide Total (MsT), direito (MsD) e esquerdo (MsE);

Cavidade Nasal Total (NCT) direita (NCD) e esquerda (NCE); Maxila Total (MxT), direita $(\mathrm{MxD})$ e esquerda (MxE);

Molar Superior Total (MST), direito (MSD) e esquerdo (MSE); Molar Inferior Total (MIT), direito (MID) e esquerdo (MIE); Mandíbula Total (MDT), direita (MDD) e esquerda (MDE); Espinha Nasal Anterior (AN), ANZ - valor zero, ANP desviado para direita, valor positivo em milímetros, ANN - desviado para a esquerda, valor negativo em milímetros.

Mento (Me), MeZ - valor zero, MeP - desviado para a direita, valor positivo em milímetros, $\mathrm{MeN}$ - desviado para a esquerda, valor negativo em milímetros (Fig. 1).

Os dados coletados foram submetidos às seguintes análises estatísticas: agrupamento de variáveis por histograma de freqüência; teste de Qui-quadrado; teste " $t$ " para dados pareados; análise de variância, modelo inteiramente casualisado; com teste de Tukey para comparação de média, com nível de significância de 5\% de probabilidade $(\mathrm{p}=0,05)$ e, cálculo de erro de mensuração segundo Midtgard et al. (1974).

\section{RESULTADO E DISCUSSÃO}

A respiração bucal é geralmente apontada como fator etiológico no desenvolvimento de deformidades transversais da face, atresias maxilares e mordidas cruzadas posteriores. Desta maneira muitos estudos têm sido desenvolvidos na tentativa de buscar uma correlação entre o padrão respiratório e a oclusão dental.

Assim, diferentemente dos estudos que abordam o envolvimento da respiração bucal com as alterações transversais, analisando, principalmente, os aspectos clínicos referentes às alterações das dimensões inter-molares, inter-caninos, da presença de mordida cruzada, este estudo pesquisou as possíveis alterações transversais dento-esqueléticas, que poderiam estar, eventualmente, relacionadas com o modo respiratório bucal.
A análise da variância (modelo inteiramente casualisado) comprovou que não existe significância estatística entre os quatro grupos p $>0,05$ (Tab. 1) para cada uma das 26 variáveis estudadas. Portanto, os indivíduos dessa amostra, com má oclusão de Classe I de Angle, na faixa etária aqui considerada, com modo respiratório bucal, não apresentaram dimensões dentofaciais transversais diferentes dos indivíduos dos demais grupos.

Uma das questões relevantes que poderia induzir a resultados diferentes de outras pesquisas na área biomédica poderia ser, sem dúvida, a faixa etária estudada. Verifica-se que existe uma relação direta da respiração bucal com a atresia maxilar e com a mordida cruzada posterior, porém, o que se nota, também, é que a faixa etária pesquisada é bastante variada e ampla. Outra questão que deve ser considerada é a variedade de má oclusão e a diversidade de padrões faciais.

O presente estudo difere, provavelmente, porque avaliou crianças com idades que variaram entre 6 anos e 1 mês a 8 anos e 2 meses, faixa etária esta que pode ser considerada estreita e baixa. Assim, nesta fase, caracterizada pelo início da dentição mista, não teria ocorrido, ainda, muitos eventos do desenvolvimento da dentição e da oclusão. Além do mais, não teria havido o surto de crescimento puberal. Poder-se-ia esperar, então, que o meio ambiente ainda não teria desencadeado esperadas alterações sobre o desenvolvimento da oclusão. Provavelmente o hábito de respirar pela boca poderia estar agindo por um curto período de tempo e sobre um padrão de desenvolvimento morfogenético facial harmonioso, como é o caso dos indivíduos desta amostra, portadores de Classe I de Angle e oclusão normal.

Por outro lado, observa-se com freqüência a descrição, na literatura, da "face adenoideana" associada a um padrão oclusal de Classe II ${ }^{31,42}$. Assim, poder-se-ia, acreditar que a presença de desarmonias esqueléticas ântero-posteriores também poderiam mascarar muitos resultados dos estudos sobre a respiração bucal. Porém, o que 
parece importante é interpretar quais seriam os efeitos da respiração bucal em indivíduos sem alterações oclusais ântero-posteriores já estabelecidas que, por si só, poderiam representar mais um componente desfavorável. Desta maneira, os resultados dos trabalhos de Linder-Aronson ${ }^{23}$, Sassouni et. al. ${ }^{38}$, Melsen et al..$^{26}$, Cheng et al. ${ }^{6}$, Naspitz et al. ${ }^{28}$, Gross et al. ${ }^{12}$ e Oulis et al. ${ }^{30}$ os quais selecionaram as amostras incluindo más oclusões variadas, encontraram diferenças estatisticamente significantes em relação às dimensões transversais dos arcos superiores entre os grupos respiração nasal e respiração bucal. Estes achados, devem ser interpretados com alguma reserva, pois poder-seia acreditar que a presença de alguma desarmonia ântero-posterior, característica da Classe II e III de Angle, já poderia propiciar alguma tendência intrínseca aos cruzamentos dentários posteriores devido à disposição mais anterior ou posterior da maxila em relação à mandíbula, induzindo a interpretações equivocadas.

Por sua vez, este estudo, ao excluir de sua

\begin{tabular}{|c|c|c|c|c|c|c|c|c|}
\hline & \multicolumn{3}{|c|}{ Efeito } & \multicolumn{3}{|c|}{ Resíduo } & \multirow[b]{2}{*}{$\mathbf{F}$} & \multirow[b]{2}{*}{$\mathbf{p}$} \\
\hline & so & GL & $\mathbf{Q M}$ & so & GL & $\mathbf{Q M}$ & & \\
\hline SphT & 25,186 & 3 & 8,395 & 672,415 & 56 & 12,007 & 0,699 & 0,557 \\
\hline SphD & 10,463 & 3 & 3,488 & 192,470 & 56 & 3,437 & 1,015 & 0,393 \\
\hline SphE & 5,769 & 3 & 1,923 & 197,965 & 56 & 3,535 & 0,544 & 0,654 \\
\hline ZygT & 54,384 & 3 & 18,128 & 1181,949 & 56 & 21,106 & 0,859 & 0,468 \\
\hline ZygD & 25,003 & 3 & 8,334 & 483,681 & 56 & 8,637 & 0,965 & 0,416 \\
\hline ZygE & 13,607 & 3 & 4,536 & 351,076 & 56 & 6,269 & 0,723 & 0,542 \\
\hline $\mathrm{MsT}$ & 97,969 & 3 & 32,656 & 1377,014 & 56 & 24,590 & 1,328 & 0,274 \\
\hline $\mathrm{MsD}$ & 47,337 & 3 & 15,779 & 445,097 & 56 & 7,948 & 1,985 & 0,127 \\
\hline $\mathrm{MsE}$ & 25,464 & 3 & 8,488 & 648,219 & 56 & 11,575 & 0,733 & 0,537 \\
\hline NCT & 1,824 & 3 & 0,608 & 186,222 & 56 & 3,325 & 0,183 & 0,908 \\
\hline NCD & 0,804 & 3 & 0,268 & 94,796 & 56 & 1,693 & 0,158 & 0,924 \\
\hline NCE & 2,537 & 3 & 0,846 & 108,709 & 56 & 1,941 & 0,436 & 0,728 \\
\hline MxT & 9,994 & 3 & 3,331 & 338,589 & 56 & 6,046 & 0,551 & 0,650 \\
\hline $\mathrm{MxD}$ & 3,142 & 3 & 1,047 & 149,504 & 56 & 2,670 & 0,392 & 0,759 \\
\hline $\mathrm{MxE}$ & 3,339 & 3 & 1,113 & 141,307 & 56 & 2,523 & 0,441 & 0,725 \\
\hline MST & 7,694 & 3 & 2,565 & 361,306 & 56 & 6,452 & 0,397 & 0,755 \\
\hline MSD & 5,570 & 3 & 1,857 & 159,864 & 56 & 2,855 & 0,650 & 0,586 \\
\hline MSE & 7,033 & 3 & 2,344 & 166,401 & 56 & 2,971 & 0,789 & 0,505 \\
\hline MIT & 9,648 & 3 & 3,216 & 282,752 & 56 & 5,049 & 0,637 & 0,594 \\
\hline MID & 1,532 & 3 & 0,511 & 160,713 & 56 & 2,870 & 0,178 & 0,911 \\
\hline MIE & 6,150 & 3 & 2,050 & 180,495 & 56 & 3,223 & 0,636 & 0,595 \\
\hline MDT & 21,529 & 3 & 7,176 & 827,455 & 56 & 14,776 & 0,486 & 0,694 \\
\hline MDD & 8,331 & 3 & 2,777 & 332,915 & 56 & 5,945 & 0,467 & 0,706 \\
\hline MDE & 2,850 & 3 & 0,950 & 435,696 & 56 & 7,780 & 0,122 & 0,947 \\
\hline AN & 0,645 & 3 & 0,215 & 45,900 & 56 & 0,820 & 0,262 & 0,852 \\
\hline $\mathrm{Me}$ & 7,692 & 3 & 2,564 & 210,054 & 56 & 3,751 & 0,684 & 0,566 \\
\hline
\end{tabular}

Análise de Variância - Modelo inteiramente casualisado.

S0 - soma do quadrado, GL - grau de liberdade, $\mathrm{QM}$ - quadrado médio, $\mathrm{F}$ - teste de distribuição, $\mathrm{p}$ - probabilidade, significância estatística quando $p<0,05$. Fonte: Curso de Odontologia da PUCPR - Curitiba-Pr, 1999. 
amostra as desarmonias ora em discussão, poderá estar trazendo informações importantes na interpretação das relações transversais dos arcos dentários com a respiração bucal, especialmente quando for considerado que não houve diferença estatisticamente significante entre os grupos estudados. É importante salientar, também, dentre as dimensões transversais mais diretamente relacionadas com a função respiratória, a dimensão transversal nasal, maxilar, molar superior, molar inferior, mandíbula, devidamente analisadas em telerradiografias póstero-anteriores, não se apresentaram significativamente diferentes nos respiração bucal quando comparadas com os respiração nasal. Desta forma, existe a probabilidade de que o padrão facial harmonioso, nesta faixa etária, poderia estar impedindo a instalação de alterações transversais significativas decorrentes do modo respiratório bucal.

Outro aspecto que parece ser relevante nesta área de estudo é a discussão quanto às metodologias adotadas para seleção da amostra. Linder-Aronson ${ }^{23}$, ${ }^{24}$, Behlfelt et al. ${ }^{2,3}$, Santos-Pinto et al. ${ }^{36}$ selecionaram as amostras de respiração bucal identificando grupos de indivíduos que apresentassem adenóides e amígdalas hipertróficas, verificando diferenças significativas quanto às dimensões transversais após um ano da remoção destas estruturas. Afirmaram, também, que haveria um retorno à normalidade do crescimento transversal maxilar e menor tendência à mordida cruzada dentária posterior. Outros, como Bresolin et $\mathrm{al} .{ }^{4}$, Naspitz et al. ${ }^{28}$, Sassouni et al. ${ }^{38}$ consideraram o grupo portador de rinite alérgica como respiração bucal, verificando diferenças significativas nas dimensões transversais dos arcos dentários entre os grupos estudados. Por outro lado Trask et al. ${ }^{40}$, também estudando um grupo de indivíduos com rinite alérgica, não encontraram diferenças significativas nas relações transversais dos arcos dentários superiores. Assim, o que poderia ser questionado, além da presença de outras variáveis, seria a ausência de outros indivíduos com respiração bucal, desde que essa não estivesse sendo causada por amígdalas e adenóide hipertróficas ou rinite alérgica.
A preocupação deste trabalho foi identificar, primeiramente, todos os indivíduos que apresentassem, efetivamente, o modo respiratório bucal, independentemente da etiologia apresentada, a partir de um universo de crianças portadoras de oclusão normal e Classe I. Pode-se afirmar, então, que o diferencial mais relevante, seria, primeiramente, a obtenção de um grupo de indivíduos efetivamente respiração bucal ${ }^{43}$ e como segundo ponto, realizar o estudo cefalométrico sem que o avaliador tivesse o conhecimento prévio dos indivíduos respiradores bucais no universo da amostra.

Uma das conseqüências da respiração bucal reportadas na literatura é a mordida cruzada dentária posterior $^{2,4,6,12,16,21,26,30,31,36}$. Os resultados do presente estudo, apontam para uma incidência muito baixa de mordida cruzada dentária posterior no grupo de respiradores bucais e que não houve diferenças estatisticamente significantes entre os grupos, quando os dados foram submetidos ao teste do Qui quadrado (Tab. 2, 3, 4). Assim, dos 60 indivíduos aleatoriamente selecionados, 40 foram identificados como respiradores bucais e 20 respiradores nasais. Deste total somente 5 indivíduos apresentaram mordida cruzada posterior, sendo 4 pertencentes ao grupo de respiradores bucais e 1 ao grupo respiração nasal. Estes resultados demonstraram que, para esta amostra, não houve correlação estatisticamente significante entre o modo respiratório bucal e o cruzamento dentário posterior.

De maneira geral, os estudos encontrados na literatura abordam as dimensões transversais das arcadas, relacionando-as com a respiração bucal. Ocorre, porém, que o diagnóstico destas atresias maxilares, arcos em "V" e mordidas cruzadas posteriores, geralmente, é realizado a partir dos aspectos clínicos ou por meio de mensurações em modelos de gesso, conforme $2,4,12,16,21,23,27,28,30,31,36$ que quantificaram as dimensões transversais das arcadas diretamente na boca com o auxílio de um paquímetro. Já Sassouni et al. ${ }^{38}$ e Cheng et al. ${ }^{6}$, realizaram as mensurações em telerradiografias póstero-anteriores. 


\begin{tabular}{|c|c|c|c|c|}
\hline \multirow[b]{2}{*}{ Grupos } & \multicolumn{4}{|c|}{ Mordida Cruzada } \\
\hline & Ausente & Direita & Bilateral & Total \\
\hline Respiração Nasal & 19 & 1 & 0 & 20 \\
\hline Respiração Bucal & 36 & 3 & 1 & 40 \\
\hline \multirow[t]{2}{*}{ Total } & 55 & 4 & 1 & 60 \\
\hline & qui-quad. & $\mathrm{GL}$ & $P$ & \\
\hline Qui-quadrado de Pearson & 0,661 & 2 & 0,718 & \\
\hline
\end{tabular}

Tabela 3 - Freqüência de indivíduos dos grupos com Oclusão - Respiração com a variável Mordida Cruzada Posterior.

\begin{tabular}{|c|c|c|c|c|}
\hline \multicolumn{2}{|c|}{ Tabela 3 - Freqüência de indivíduos dos grupos com Oclusão - Respiração com a variável Mordida Cruzada Posterior. } \\
\hline Grupos & \multicolumn{2}{|c|}{ Mordida Cruzada } \\
\hline Oclusão Normal - Respiração Nasal & Ausente & Direita & Bilateral & Total \\
\hline Oclusão Normal - Respiração Bucal & 14 & 0 & 0 & $\mathbf{1 4}$ \\
\hline Classe I- Respiração Nasal & 11 & 0 & 0 & $\mathbf{1 1}$ \\
\hline Classe I - Respiração Bucal & 5 & 1 & 0 & $\mathbf{6}$ \\
\hline Total & 25 & 3 & 1 & $\mathbf{2 9}$ \\
\hline
\end{tabular}

\begin{tabular}{|c|c|c|c|c|}
\hline \multirow[b]{2}{*}{ Grupos } & \multicolumn{4}{|c|}{ Mordida Cruzada } \\
\hline & Ausente & Direita & Bilateral & Total \\
\hline Classe I - Respiração Nasal & 5 & 1 & 0 & 6 \\
\hline Classe I - Respiração Bucal & 25 & 3 & 1 & 29 \\
\hline \multirow[t]{2}{*}{ Total } & 30 & 4 & 1 & 35 \\
\hline & qui-Quad. & GL & $\mathrm{p}$ & \\
\hline Qui quadrado de Pearson & 0,386 & 2 & 0,824 & \\
\hline
\end{tabular}

Por outro lado pode-se afirmar que com o avanço tecnológico há necessidade de se realizar um diagnóstico mais preciso, utilizando-se de elementos complementares mais adequados. Desta forma, a escolha de pontos de referências cranianos confiáveis apresenta-se como um primeiro e maior obstáculo. Neste particular, Ricketts ${ }^{33,35}$ utilizou-se de pontos localizados no arco zigomático, esfenóide e septo nasal. Já Chierici ${ }^{7}$ propôs o arco zigomático e crista galli como referenciais. Ainda que o etmóide seja um referencial craniano im- portante, a variabilidade do zigomático, e, principalmente, do septo nasal pode ser questionada. $\mathrm{Da}$ mesma forma, o plano orbital, construído a partir de pontos de referência localizados na própria órbita, com intuito de avaliar a variabilidade horizontal e vertical das estruturas faciais, conforme as propostas de Sassouni ${ }^{37}$, Cheney ${ }^{5}$, Letzer e Kronman $^{20}$, Faber ${ }^{9}$, deve ser considerado com ressalvas. Por sua vez, a espinha nasal anterior, sugerida por Grummons e Van de Copello ${ }^{13}$, apresenta dificuldade similar, pois é de domínio geral a grande va- 
riabilidade espacial da maxila em relação à base craniana e demais estruturas faciais. A proposta de Grayson e Bookstein ${ }^{10}$, que se apoia em três linhas medianas, cada uma delas localizada em diferentes profundidades do complexo craniofacial, tem a sua utilidade, principalmente no estudo das relações das estruturas faciais localizadas em diferentes profundidades do complexo craniofacial. Porém, para os propósitos deste trabalho, pelas dificuldades de interpretação e por ser pouco prática para a rotina do diagnóstico ortodôntico, esta proposta não foi considerada.

Portanto, por acreditar-se que, para a correta avaliação das dimensões transversais da face, especialmente para a análise comparativa entre medidas lineares horizontais ou verticais das hemi faces direita e esquerda, torna-se imprescindivel a adoção de referenciais cranianos confiáveis para a construção de uma linha média facial verdadeira. Assim, a escolha dos pontos craniométricos localizados em estruturas ósseas estáveis que, preferencialmente, não sofram influências negativas extrínsecas durante o seu crescimento deve ser o principal pré-requisito. Neste particular, Pearson e Woo ${ }^{32}$ já descreviam o osso esfenóide como sendo uma das estruturas craniofaciais mais estáveis e simétricas. Considerandose a ausência na literatura de propostas de pontos craniométricos apoiados na premissa aqui discutida, é que optou-se, neste trabalho, pela escolha dos pontos esfenóide direito e esfenóide esquerdo, definidos como referenciais para a proposta da linha esfenoidal horizontal (LEH).
Assim, os resultados deste estudo comprovaram a confiabilidade da metodologia empregada, ao se utilizar desta linha de referência. A análise estatística demonstrou que o osso esfenóide, nessa amostra, apresentou elevado grau de simetria $\mathrm{p}=0,714$ (Tab. 5). Por conseguinte, tornou-se viável o emprego de uma linha média sagital verdadeira que, efetivamente, representasse a divisão da face humana ao meio, a partir da crista galli e desde que intersectasse ortogonalmente a linha LEH.

Por outro lado para as demais medidas: zigomático, mastóide, cavidade nasal, maxila, molar superior, molar inferior e mandíbula, os resultados apresentaram-se estatisticamente significantes $\mathrm{p}<0,05$ (Tab. 6, 7, 8, 9, 10, 11, 12), ou seja, existe uma assimetria entre os lados direito e esquerdo caracterizada, sempre, por apresentar o lado direito significativamente maior que o esquerdo.

A hipótese de que a LMV efetivamente seria representativa e útil, confirmou-se até mesmo para o ponto Me, ponto mais distante da base craniana, que apresentou uma variabilidade para os lados direito e esquerdo de 1,92 mm. A mesma interpretação deve ser considerada para a variável AN, $(0,89 \mathrm{~mm}$ de desvio padrão) que, na prática, representa eventuais rotações da maxila para a direita ou para a esquerda.

Este estudo restringiu as suas observações a um grupo de crianças com harmonia facial ântero-posterior e transversal e com faixa etária compreendida entre 6 e 8 anos de idade. Recomenda-se estudos complementares em outras faixas etárias com más oclusões distintas deste trabalho.

\begin{tabular}{|c|c|c|c|c|c|c|c|c|c|}
\hline & Média & Desv. pad. & $\mathbf{N}$ & Dif. méd. & Desv. pad. das dif. & to & GL & $\mathbf{p}$ & CV (\%) \\
\hline SphD & 45,133 & 1,855 & & & & & & & 4,1 \\
\hline SphE & 45,067 & 1,858 & 60 & 0,067 & 1,401 & 0,369 & 59 & 0,714 & 4,1 \\
\hline \multicolumn{10}{|c|}{ Tabela 6 - Análise comparativa entre zigomático, lado direito e esquerdo. } \\
\hline & Média & Desv. pad. & $\mathbf{N}$ & Dif. méd. & Desv. pad. das dif. & to & GL & $\mathbf{p}$ & CV (\%) \\
\hline ZygD & 59,117 & 2,936 & & & & & & & 4.9 \\
\hline ZygE & 56,717 & 2,486 & 60 & 2,4 & 2,941 & 6,321 & 59 & 0,000 & 4.3 \\
\hline
\end{tabular}




\begin{tabular}{|c|c|c|c|c|c|c|c|c|c|}
\hline & Média & Desv. pad. & $\mathbf{N}$ & Dif. méd. & Desv. pad. das dif. & to & GL & $\mathbf{p}$ & CV (\%) \\
\hline $\mathrm{MsD}$ & 54,633 & 2,889 & & & & & & & 5.3 \\
\hline MsE & 50,383 & 3,379 & 60 & 4,25 & 3,812 & 8,636 & 59 & 0,000 & 6.7 \\
\hline \multicolumn{10}{|c|}{ Tabela 8 - Análise comparativa entre cavidade nasal, lado direito e esquerdo. } \\
\hline & Média & Desv. pad. & $\mathbf{N}$ & Dif. méd. & Desv. pad. das dif. & to & GL & p & CV (\%) \\
\hline NCD & 14,300 & 1,273 & & & & & & & 8.9 \\
\hline NCE & 13,258 & 1,373 & 60 & 1,04 & 1,956 & 4,126 & 59 & 0,000 & 10.3 \\
\hline
\end{tabular}

\begin{tabular}{|c|c|c|c|c|c|c|c|c|c|}
\hline & Média & Desv. pad. & $\mathbf{N}$ & Dif. méd. & Desv. pad. das dif. & to & GL & $\mathbf{p}$ & CV (\%) \\
\hline $\mathrm{MxD}$ & 30,708 & 1,608 & & & & & & & 5.2 \\
\hline MxE & 29,708 & 1,566 & 60 & 1,00 & 2,042 & 3,793 & 59 & 0,000 & 5.2 \\
\hline
\end{tabular}

\begin{tabular}{|c|c|c|c|c|c|c|c|c|c|}
\hline \multicolumn{9}{|c|}{ Tabela 10 } & Análise comparativa entre molar superior, lado direito e esquerdo. \\
\hline & Média & Desv. pad. & N & Dif. méd. & Desv. pad. das dif. & to & GL & p & CV (\%) \\
\hline MSD & 28,967 & 1,675 & & & & & & 5.7 \\
\hline MSE & 27,533 & 1,715 & 60 & 1,43 & 2,288 & 4,854 & 59 & 0,000 & 6.2 \\
\hline
\end{tabular}

\begin{tabular}{|c|c|c|c|c|c|c|c|c|c|}
\hline & Média & Desv. pad. & $\mathbf{N}$ & Dif. méd. & Desv. pad. das dif. & to & $\mathbf{G L}$ & $\mathbf{p}$ & CV (\%) \\
\hline MID & 28,408 & 1,658 & & & & & & & 5.8 \\
\hline MIE & 27,208 & 1,779 & 60 & 1,2 & 2,628 & 3,536 & 59 & 0,001 & 6.5 \\
\hline \multicolumn{10}{|c|}{ Tabela 12 - Análise comparativa entre mandíbula, lado direito e esquerdo. } \\
\hline & Média & Desv. pad. & $\mathbf{N}$ & Dif. méd. & Desv. pad. das dif. & to & GL & $\mathbf{p}$ & CV (\%) \\
\hline MDD & 39,842 & 2,405 & & & & & & & 6.0 \\
\hline MDE & 37,808 & 2,726 & 60 & 2,03 & 3,194 & 4,931 & 59 & 0,000 & 7.2 \\
\hline
\end{tabular}

Teste t para dados pareados (quando duas medidas são fornecidas pelo mesmo indivíduo). Desv. pad. - desvio padrão da amostra; $\mathbf{N}$ - tamanho da amostra; Dif. méd. - diferenças entre as médias das amostras; Desv. pad. das dif. - desvio padrão das diferenças entre amostras; to - valor do teste t; GL - graus de liberdade; $\mathbf{p}$ - probabilidade de erro; CV (\%) - coeficiente de variação.

As diferenças estatísticas entre os dois grupos são assinalados por $\mathrm{p}<0,05$.

\section{CONCLUSÃO}

Os indivíduos com má oclusão Classe I com respiração bucal apresentaram dimensões transversais similares ao grupo com respiração nasal. Nesta amostra, a atresia maxilar não está relacionada aos indivíduos respiradores bucais; a ca- vidade nasal não se mostrou diminuída no grupo de respiradores bucais; a distância inter-molares superiores no grupo de respiração bucal não foi diferente do grupo de respiração nasal; a mordida cruzada posterior não é uma característica clínica indicativa de respiração bucal. 
A study of facial transverse dimensions, in postero-anterior teleradiography in mouth breather individuals with normal occlusion and malloclusion Classe I of Angle

\begin{abstract}
Mouth breathing has been studied for more than 100 years, trying to search the real relationship with the malocclusions. The aim of this study is to assess the transversal facial dimensions of 60 children: 32 females and 28 males, whose had Angle Class I malocclusion with mouth and nasal breathing. 60 teleradiographies were outlined and selected in posteroanterior frontal norm to attainment of the 26 variables to the study. The evaluation of the respiratory pattern of each child was realized by a visual examination, to register the labial posture and, by a questionnaire answered by the parents of the child, which had data concerning medical history and respiratory pattern. After the diagnosis of respiratory mode, the sample was divided in groups: normal occlusion with nasal breathing (14 children), normal occlusion with mouth breathing (11 children), Angle Class I with nasal breathing ( 6 children), Angle Class I with mouth breathing (29 children). The results indicate that are no statistical significantly differences in the transversal dimensions of the face between the groups, that means, in this sample the individuals with Class I malocclusion with mouth breathing, showed transversal dimensions similar to the group with nasal breathing. Furthermore, no correlations were founded between the mouth breathing mode and posterior crossbite.
\end{abstract}

Key words: Mouth Breathing. Posteroanterior cephalometric. Diagnosis.

\title{
REFERÊNCIAS
}

1. ANGLE, E. H. Classification of malocclusion. Dent Cosmos, Philadelphia, p. 248-264, 1898.

2. BEHLFELT, K.; LINDER-ARONSON, S.; McWilliams, J. et al. Dentition in children with enlarged tonsils compared to control children. Eur J Orthod, London, v. 11, p. 416-429, 1989.

3. BEHLFELT, K. Enlarged tonsils and the effect of tonsillectomy: characteristics of the dentition and facial skeleton, posture of the head, hyoid bone and tongue, mode of breathing. Swed Dent J, Stockholm, v. 72, p. 1-35, 1990. Supplement.

4. BRESOLIN, D.; SHAPIRO, P. A.; SHAPIRO, G. G. et al. Mouth breathing in allergic children: its relationship to dentofacial development. Am J Orthod, St. Louis, v. 83, no. 4, p. 334-340, Apr. 1983

5. CHENEY, E. A. Dentofacial asymmetries and their clinical significance. Am J Orthod, St. Louis, v. 47, no. 11, p. 814-829, Nov. 1961.

6. CHENG, M. C.; ENLOW, D. H.; PAPSIDERO, M. et al. Developmental effects of impaired breathing in the face of the growing child. Angle Orthod, Appleton, v. 58, no. 4, p. 309-320, Oct. 1988.

7. CHIERICl, G. Radiologic assessment of facial asymmetry. In: HARVOLD, E. P. (Ed.). Treatment of hemifacial mikrosomia. New York: Alan R. Liss, 1983. p. 57-87.

8. EMSLIE, R. D.; MASSLER, M.; ZWEMER, J. D. Mouth breathing: etiology and effects (a review). J Am Dent Assoc, Chicago, v. 44, p. 506-521, May 1952.

9. FABER, R. D. The differential diagnosis and treatment of crossbites. Dent Clin North Am, Philadelphia, v. 25, no.1, p. 53-68, Jan. 1981.

10. GRAYSON, B. H.; BOOKSTEIN, F. Analysis of craniofacial asymmetry by multiplane cephalometry. Am J Orthod Dentofacial
Orthop, St. Louis, v. 84, no. 3, p. 217-224, Sept. 1983.

11. GROSS, A. G.; KELLUM, G. D.; FRANZ, D. et al. A longitudinal evaluation of open mouth posture and maxillary arch with in children. Angle Orthod, Appleton, v. 64, no. 6, p. 419-424, Jan. 1994.

12. GROSS, A. G.; KELLUM, G. D.; FRANZ, D. et al. Open-mouth posture and maxillary arch width in young children: a three-year evaluation. Am J Orthod Dentofacial Orthop, St. Louis, v. 106, no. 6, p. 635-640, Dec. 1994.

13. GRUMMONS, D. C.; VAN DE COPPELLO, K. A frontal asymmetry analysis. Clin Orthod, Boulder, v. 21, p. 448-465, July 1987.

14. GWYNNE-EVANS, E. Discussion on the mouth-breather. Proc $\mathbf{R}$ Soc Med, London, v. 51, p. 279-285, Oct. 1957.

15. GWYNNE-EVANS, E.; BALLARD, C. F. The mouth breather. Am J Orthod, St. Louis, v. 44, p. 559, July 1958.

16. HANNUKSELA, A.; VÄÄNÄNEN, A. Predisposing factors for malocclusion in 7-year-old children with special reference to a topic diseases. Am J Orthod Dentofacial Orthop, St. Louis, v. 92, no. 4, p. 299-303, Oct. 1987

17. HARTSOOK, J. T. Mouth breathing as a primary etiologic factor in the production of malocclusion. J Dent Child, Chicago, v.13, p. 91-94, 1946

18. HAWKINS, A. C. Mouth breathing and its relationship to malocclusion and facial abnormalities, New Mexico Dent J, Mexico, DC, v. 20, no.1, p.18-21, May 1969.

19. KINGSLEY, W. S. A Treatise on oral deformities as a branch of mechanical surgery. New York: Appleton, 1889.

20. LETZER, G. M.; KRONMAN, J. H. A posteroanterior cephalometric evaluation of craniofacial asymmetry. ,Appleton, v. 37, no. 3, p. 205-211, July 1967.

21. LINDER-ARONSON, S. BÄCKSTRÖM, A. A comparison betwe- 
en mouth and nose breathers with respect to occlusion and facial dimensions. Odontol Revy, Sweden, v.11, no. 4, p. 343376, 1960.

22. LINDER-ARONSON, S. Dimensions of face and palate in nose breathers and in habitual mouth breathers. Odontol Revy, Sweden, v.14, no. 2, p.187-199, Feb. 1963.

23. Effects of adenoidectomy on dentition and nasopharynx. Am J Orthod, St. Louis, v. 65, no. 1, p. 1-15, Jan. 1974.

24. Respiratory function in relation to facial morphology and the dentition. Br J Orthod, London, v. 6, no. 2, p. 59-71, Apr. 1979.

25. McNAMARA Jr., J. A. Influence of respiratory patterespiração nasal on craniofacial growth. Angle Orthod, Appleton, v. 51, no. 4, p. 269-300, Oct. 1981.

26. MELSEN, B.; ATTINA, L.; SANTUARI, M. et al. Relationship between swallowing patterespiração nasal, mode of respiration, and development of malocclusion. Angle Orthod, Appleton, v. 57, no. 2, p.113-120, Apr. 1987.

27. MOREIRA, M. Avaliação da profundidade e dimensões transversais do palato em indivíduos respiradores bucais, na dentição decídua. Rev Paul Odontol, São Paulo, v.11, n. 5, p. 20-30, set./ out. 1989.

28. NASPITZ, N. R. L. L.; FALTIN, K. J. et al. Estudo das medidas transversais dos maxilares em pacientes respiradores bucais e nasais portadores de maloclusões dentárias. Rev Bras Alerg Imunopatol, São Paulo, v. 13, p. 226-228, 1990.

29. O'RYAN, F. S. The relation between nasorespiratory function and dentofacial morphology: a review. Am J Orthod Dentofacial Orthop, St. Louis, v. 82, no. 5, p. 403-410, Nov. 1982.

30. OULIS, C. J.; VADIAKAS, G. P.; EKONOMIDES, J. et al. The effect of hypertrophic adenoids and tonsils on the development of posterior cross bite and oral habits. J Clin Pediatr Dent, Birmingham, v.18, no. 3, p.197-201,1994.

31. PAUL, J. L.; NANDA, R. S. Effect of mouth breathing on dental occlusion. Angle Orthod, Appleton, v. 43, no. 2, p. 201-206, Apr. 1973.

32. PEARSON, K.; WOO, T. L. Further investigation of the morphometric characters of the individual bones of the human skull. Biometrika, Cambridge, v. 27, p. 424-465, Oct. 1935.
33. RICKETTS, R. M BENCH, R. W.; HILGERS, J. J. et al. An overview of computerized cephalometrics. Am J Orthod, St. Louis, v. 61, no.1, p.1-28, Jan. 1972

34. RICKETTS, R. M. Respiratory obstruction syndrome. Am J Orthod, St. Louis, v. 54, no. 7, p. 495-507, July 1968.

$35 . \quad$ Perspectives in the clinical application of cephalometrics. Angle Orthod, Appleton, v. 51, no. 2, p.115-150, Apr. 1981.

36. SANTOS-PINTO, C. C. M.; HENRIQUES, J. F. C.: PINZAN, A et al. Estudo radiográfico e de modelos, para a avaliação de alterações dentofaciais em função da redução do espaço nasofaringeano em jovens brasileiros leucodermas de 8 a 14 anos de idade. Ortodontia, São Paulo, v. 26, n. 2, p. 57-74, maio/ago. 1993.

37. SASSOUNI, V. Diagnosis and treatment planning via roentgenographic cephalometry. Am J Orthod, St. Louis, v. 44, no. 6, p. 433-463, June 1958.

38. SASSOUNI, V.; FRIDAY, G. A.; SHNORHOKIAN, H. et al. The influence of perennial allergic rhinitis on facial type and a pilot study of the effect of allergy management on facial growth patterespiração nasals. Ann Allergy, Minneapolis, v. 54, p. 493497, June 1985.

39. SHAUGHNESSY, T. G. The relationship between upper airway obstruction and craniofacial growth. J Mich Dent Assoc, Lansing, v. 65, p. 431-433, Sept. 1983.

40. TRASK, G. M.; SHAPIRO, G. G.; SHAPIRO, P. A. The effects of perennial allergic rhinitis on dental and skeletal development: $A$ comparison of sibling pairs. Am J Orthod Dentofacial Orthop, St. Louis, v. 92, no. 4, p. 286-293, Oct. 1987.

41. TOMES, C. S. The bearing of the development of the jaws on irregularities. Dent Cosmos, Philadelphia, v.15, p. 292-296, Feb. 1873.

42. VIG, K. W. L. Nasal Obstruction and facial growth: the strength of evidence for clinical assumptions. Am J Orthod Dentofacial Orthop, St. Louis, v. 113, no. 6, p. 603-611, June 1998.

43. WAGNITZ, S. J. V. Diagnóstico do modo respiratório. 2000. Dissertação (Mestrado em Odontologia)-Pontifícia Universidade Católica do Paraná, Curitiba, 2000.

\footnotetext{
Endereço para correspondência

Rosemári Fistarol Daniel

Rua Guilherme Pugsley, 1551, apto. 43 - Água Verde

CEP: $80620-000$

Curitiba - PR

e-mail: rosemaridaniel@onda.com.br
} 